\title{
Improved undetected error probability model for JTEC and JTEC-SQED coding schemes
}

\begin{abstract}
The undetected error probability is an important measure to assess the communication reliability provided by any error coding scheme. Two error coding schemes namely, Joint crosstalk avoidance and Triple Error Correction (JTEC) and JTEC with Simultaneous Quadruple Error Detection (JTEC-SQED), provide both crosstalk reduction and multi-bit error correction/detection features. The available undetected error probability model yields an upper bound value which does not give accurate estimation on the reliability provided. This paper presents an improved mathematical model to estimate the undetected error probability of these two joint coding schemes. According to the decoding algorithm the errors are classified into patterns and their decoding result is checked for failures. The probabilities of the failing patterns are used to build the new models. The improved models have less than $1 \%$ error with respect to the simulation results and reflect in up to $60 \%$ higher mean time to failure as compared to available models.
\end{abstract}

Keyword: Error control; Fault tolerance; Crosstalk; On chip interconnects; Error probability 